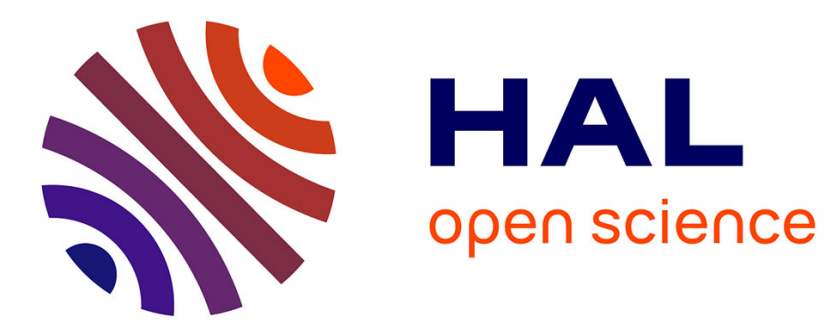

\title{
On the flow of a viscoplastic fluid in a thinperiodic domain
}

\author{
María Anguiano, Renata Bunoiu
}

\section{To cite this version:}

María Anguiano, Renata Bunoiu. On the flow of a viscoplastic fluid in a thinperiodic domain. Integral Methods in Science and Engineering: Analytic Treatment and Numerical Approximations, Birkhäuser, pp.15-24, 2019, 978-3-030-16076-0. 10.1007/978-3-030-16077-7_2 . hal-03264746

\section{HAL Id: hal-03264746 \\ https://hal.science/hal-03264746}

Submitted on 9 Jan 2022

HAL is a multi-disciplinary open access archive for the deposit and dissemination of scientific research documents, whether they are published or not. The documents may come from teaching and research institutions in France or abroad, or from public or private research centers.
L'archive ouverte pluridisciplinaire HAL, est destinée au dépôt et à la diffusion de documents scientifiques de niveau recherche, publiés ou non, émanant des établissements d'enseignement et de recherche français ou étrangers, des laboratoires publics ou privés. 


\title{
On the flow of a viscoplastic fluid in a thin periodic domain
}

\author{
María Anguiano and Renata Bunoiu
}

\subsection{Introduction}

We study in this paper the steady incompressible nonlinear flow of a Bingham fluid in a thin periodic domain, which is a model of porous media. The model of thin porous media of thickness much smaller than the parameter of periodicity was introduced in [Zh08], where a stationary incompressible Navier-Stokes flow was studied. Recently, the model of the thin porous medium under consideration in this paper was introduced in [FaEtAl16], where the flow of an incompressible viscous fluid described by the stationary Navier-Stokes equations was studied by the multiscale asymptotic expansion method, which is a formal but powerful tool to analyse homogenization problems. These results were rigorously proved in [AS18] using an adaptation (introduced in [AS17]), of the unfolding method from [CiEtA108]. This adaptation consists of a combination of the unfolding method with a rescaling in the height variable, in order to work with a domain of fixed height, and to use monotonicity arguments to pass to the limit. In [AS17], in particular, the flow of an incompressible stationary Stokes system with a nonlinear viscosity, being a power law, was studied. For nonstationary incompressible viscous fluid flow in a thin porous medium we refer to [An17], where a nonstationary Stokes system is considered, and [An217], where a nonstationary non-Newtonian Stokes system, where the viscosity obeys the power law, is studied. For the unfolding method applied to the study of problems stated in other type of thin periodic domains we refer for instance to [Gr04] for crane type structures and to [GrEtA117] for thin layers with thin beams structures, where elasticity problems are studied.

M. Anguiano

Universidad de Sevilla, Sevilla, Spain,

e-mail: anguiano@us.es

R. Bunoiu

Université de Lorraine, Metz, France, e-mail: renata.bunoiu@univ-loraine.fr 
Viscoplastic fluids are quite often encountered in real life. We mention oils, polymer solutions, vocanic lavas, muds and clays, avalanches, liquid chocolate. The theory of the fluid mechanics of such materials has several different applications, as for instance in the oil and gas industry, which can be found in the ground, which is a porous medium. The most commonly studied viscoplastic fluid is the Bingham fluid. In our thin porous medium, we consider the flow of a nonlinear viscoplastic Bingham flow, whose yield stress itself depends on the small parameter characterizing the geometry of the domain, denoted $\varepsilon$. The first study of this type of problem is due to [LiEtAl81], where the problem was studied in a classical porous medium, by using the multiscale asymptotic expansion method. A nonlinear Darcy law was obtained after the passage to the limit $\varepsilon \rightarrow 0$. The corresponding convergence result was proved in [BoEtA193] with the two-scale convergence method and then recovered in [BuEtAl13] with the periodic unfolding method from [CiEtAl08]. For the study in a porous medium with a doubly periodic structure we refer to [BuEtAl17], where a more involved nonlinear Darcy law was derived. The flow of a Bingham fluid was also studied in thin domains of small height, denoted $\varepsilon$. We refer the reader to [BuEtAl03], [BuEtA104], and [BuEtAl18] for these studies, where a lower dimensional Bingham-like law was exhibited from the limit problem, after the passage to the limit $\varepsilon \rightarrow 0$. This law was already used in the engineering (see [LiEtA190]), but no rigourous mathematical derivation was previously known.

The paper is organized as follows. In Section 1.2 we state the problem: we define in (1.1) the thin porous medium (see also Fig. 1.3), in which we consider the flow of a viscoplastic Bingham fluid with velocity verifying the nonlinear variational inequality (1.5). In Section 1.3 we state and prove the main result of our paper, Theorem 1. We then give in Section 1.4 some conclusions and perspectives and we end the paper with a list of References.

\subsection{Statement of the Problem}

The domain: the periodic porous medium is defined by a domain $\omega$ and an associated microstructure, or periodic cell $Y^{\prime}=[-1 / 2,1 / 2]^{2}$, which is made of two complementary parts: the fluid part $Y_{f}^{\prime}$, and the solid part $Y_{s}^{\prime}\left(Y_{f}^{\prime} \cup Y_{s}^{\prime}=Y^{\prime}\right.$ and $\left.Y_{f}^{\prime} \cap Y_{s}^{\prime}=\varnothing\right)$. More precisely, we assume that $\omega$ is a smooth, bounded, connected set in $\mathbb{R}^{2}$, and that $Y_{s}^{\prime}$ is an open connected subset of $Y^{\prime}$ with a smooth boundary $\partial Y_{s}^{\prime}$, such that $\bar{Y}_{s}^{\prime}$ is strictly included in $Y^{\prime}$.

The microscale of the porous medium is a small positive number $\varepsilon$. The domain $\omega$ is covered by a regular mesh of square of size $\varepsilon$ : for $k^{\prime} \in \mathbb{Z}^{2}$, each cell $Y_{k^{\prime}, \varepsilon}^{\prime}=\varepsilon k^{\prime}+\varepsilon Y^{\prime}$ is divided in a fluid part $Y_{f^{\prime}, \varepsilon}^{\prime}$ and a solid part $Y_{s_{k^{\prime}}, \varepsilon}^{\prime}$, i.e. is similar to the unit cell $Y^{\prime}$ rescaled to size $\varepsilon$. We define $Y=Y^{\prime} \times(0,1) \subset \mathbb{R}^{3}$, which is divided in a fluid part $Y_{f}=Y_{f}^{\prime} \times(0,1)$ and a solid part $Y_{s}=Y_{s}^{\prime} \times(0,1)$, and consequently 
$Y_{k^{\prime}, \varepsilon}=Y_{k^{\prime}, \varepsilon}^{\prime} \times(0,1) \subset \mathbb{R}^{3}$, which is divided in a fluid part $Y_{f_{k^{\prime}}, \varepsilon}$ and a solid part $Y_{s_{k^{\prime}}, \varepsilon}$.

We define $\Lambda_{\varepsilon}$ (see Fig. 1.1) by

$$
\Lambda_{\varepsilon}=\omega \times(0, \varepsilon) .
$$

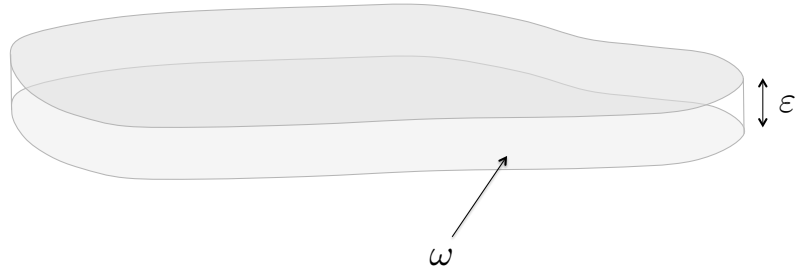

Fig. 1.1 Views of the domain $\Lambda_{\varepsilon}$

We denote by $\tau\left(\bar{Y}_{s_{k^{\prime}}, \varepsilon}^{\prime}\right)$ the set of all translated images of $\bar{Y}_{s_{k^{\prime}}, \varepsilon}^{\prime}$. The set $\tau\left(\bar{Y}_{s_{k^{\prime}}, \varepsilon}^{\prime}\right)$ represents the solids in $\mathbb{R}^{2}$. The fluid part of the bottom $\omega_{\varepsilon} \subset \mathbb{R}^{2}$ of the porous medium is defined by $\omega_{\varepsilon}=\omega \backslash \bigcup_{k^{\prime} \in \mathscr{K}_{\varepsilon}} \bar{Y}_{s_{k^{\prime}}, \varepsilon}^{\prime}$ (see Fig. 1.2), where $\mathscr{K}_{\varepsilon}=\left\{k^{\prime} \in \mathbb{Z}^{2}\right.$ : $\left.Y_{k^{\prime}, \varepsilon}^{\prime} \cap \omega \neq \emptyset\right\}$. The whole fluid part $\Omega_{\varepsilon} \subset \mathbb{R}^{3}$ in the thin porous medium is defined by

$$
\Omega_{\varepsilon}=\left\{\left(x_{1}, x_{2}, x_{3}\right) \in \omega_{\varepsilon} \times \mathbb{R}: 0<x_{3}<\varepsilon\right\} .
$$

We make the assumption that the solids $\tau\left(\bar{Y}_{s_{k^{\prime}}, \varepsilon}^{\prime}\right)$ do not intersect the boundary $\partial \omega$ (see Fig. 1.2):

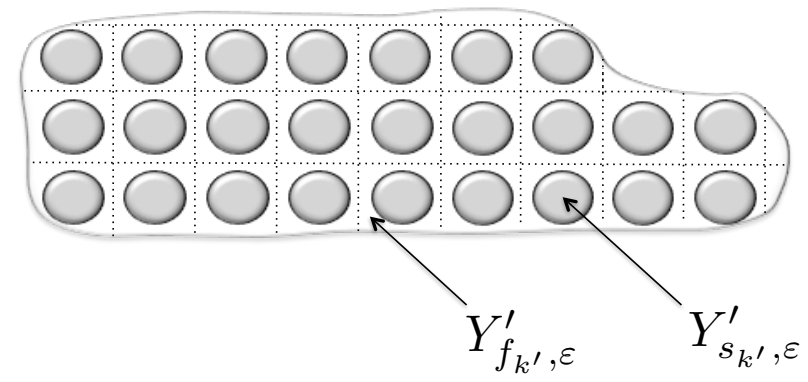

Fig. 1.2 Views of the domain $\omega_{\varepsilon}$

We define $Y_{s_{k^{\prime}}, \varepsilon}^{\varepsilon}=Y_{s_{k^{\prime}}, \varepsilon}^{\prime} \times(0, \varepsilon)$. Denote by $S_{\varepsilon}$ the set of the solids contained in $\Omega_{\varepsilon}$ (see Fig. 1.3). Then, $S_{\varepsilon}$ is a finite union of solids, i.e.

$$
S_{\varepsilon}=\bigcup_{k^{\prime} \in \mathscr{K}_{\varepsilon}} \bar{Y}_{s_{k^{\prime}}, \varepsilon}^{\varepsilon}
$$




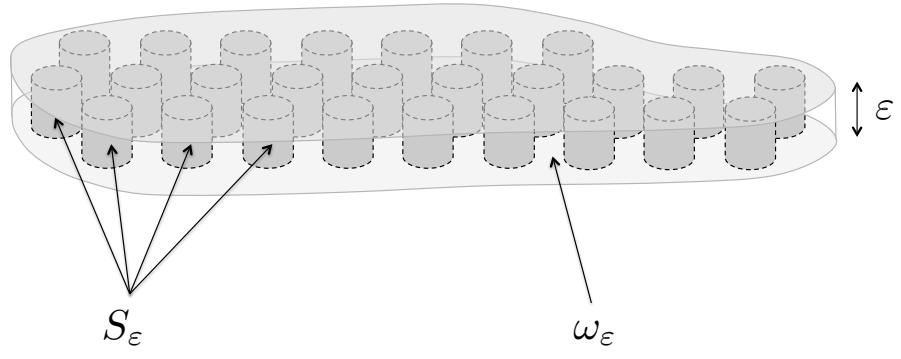

Fig. 1.3 Views of the domain $\Omega_{\varepsilon}$

We define

$$
\widetilde{\Omega}_{\varepsilon}=\omega_{\varepsilon} \times(0,1), \quad \Omega=\omega \times(0,1) .
$$

We observe that $\widetilde{\Omega}_{\varepsilon}=\Omega \backslash \bigcup_{k^{\prime} \in \mathscr{K}_{\varepsilon}} \bar{Y}_{s_{k^{\prime}}, \varepsilon}$, and we define $T_{\varepsilon}=\bigcup_{k^{\prime} \in \mathscr{K}_{\varepsilon}} \bar{Y}_{s_{k^{\prime}}, \varepsilon}$ as the set of the solids contained in $\widetilde{\Omega}_{\varepsilon}$.

The problem: in the domain $\Omega_{\varepsilon}$ defined in (1.1), we consider the stationary flow of an incompressible Bingham fluid. Following [DuEtA172], the problem is formulated in terms of a nonlinear variational inequality.

For a vectorial function $v$, we define $(1 \leq i, j \leq 3)$

$$
(D(v))_{i, j}=\frac{1}{2}\left(\partial_{x_{j}} v_{i}+\partial_{x_{i}} v_{j}\right), \quad|D(v)|^{2}=D(v): D(v)
$$

where : denotes the full contraction of two matrices: for $A=\left(a_{i, j}\right)_{1 \leq i, j \leq 3}$ and $B=$ $\left(b_{i, j}\right)_{1 \leq i, j \leq 3}$, we have $A: B=\sum_{i, j=1}^{3} a_{i j} b_{i j}$.

We consider the space

$$
V\left(\Omega_{\varepsilon}\right)=\left\{v \in\left(H_{0}^{1}\left(\Omega_{\varepsilon}\right)\right)^{3} \mid \operatorname{div} v=0 \text { in } \Omega_{\varepsilon}\right\},
$$

and for $u, v \in\left(H_{0}^{1}\left(\Omega_{\varepsilon}\right)\right)^{3}$, we introduce

$$
\begin{gathered}
a(u, v)=2 \mu \int_{\Omega_{\varepsilon}} D(u): D(v) d x, \\
j(v)=\sqrt{2} g \varepsilon \int_{\Omega_{\varepsilon}}|D(v)| d x, \\
(u, v)_{\Omega_{\varepsilon}}=\int_{\Omega_{\varepsilon}} u \cdot v d x
\end{gathered}
$$

where the positive real $\mu$ is the viscosity of the Bingham fluid and the positive real $g$ is related to the yield stress of the Bingham fluid. More precisely, the yield stress of the Bingham fluid under consideration in this work is of the form $g \varepsilon$, where $\varepsilon$ is the parameter related to the geometry of the domain. This yield stress is exactly the one considered by [LiEtA181], where the flow of a Bingham fluid in a classical porous medium was studied. 
Let $f \in\left(L^{2}(\Omega)\right)^{3}$ be given such that $f=\left(f^{\prime}, 0\right)$ and $f_{\varepsilon} \in\left(L^{2}\left(\Omega_{\varepsilon}\right)\right)^{3}$ be defined by

$$
f_{\mathcal{\varepsilon}}(x)=f\left(x^{\prime}, x_{3} / \varepsilon\right), \text { a.e. } x \in \Omega_{\varepsilon} .
$$

The model of the flow is described by the following nonlinear variational inequality:

Find $u_{\varepsilon} \in V\left(\Omega_{\varepsilon}\right)$ such that

$$
a\left(u_{\varepsilon}, v-u_{\varepsilon}\right)+j(v)-j\left(u_{\varepsilon}\right) \geq\left(f_{\varepsilon}, v-u_{\varepsilon}\right) \Omega_{\varepsilon}, \quad \forall v \in V\left(\Omega_{\varepsilon}\right) .
$$

From [DuEtA172], we know that for every fixed $\varepsilon$ there exists a unique $u_{\varepsilon} \in$ $V\left(\Omega_{\varepsilon}\right)$ solution of problem (1.5).

\subsection{Main Convergence Result}

Our aim is to study the asymptotic behavior of $u_{\mathcal{E}}$, solution of problem (1.5), when $\varepsilon$ tends to zero. For this purpose, we first use the dilatation of the domain $\Omega_{\varepsilon}$ in the variable $x_{3}$, namely

$$
y_{3}=\frac{x_{3}}{\varepsilon},
$$

in order to have the functions defined in an open set with fixed height, denoted $\widetilde{\Omega}_{\varepsilon}$ and given by (1.2).

Namely, we define $\tilde{u}_{\varepsilon} \in\left(H_{0}^{1}\left(\widetilde{\Omega}_{\varepsilon}\right)\right)^{3}$ by

$$
\tilde{u}_{\varepsilon}\left(x^{\prime}, y_{3}\right)=u_{\varepsilon}\left(x^{\prime}, \varepsilon y_{3}\right) \text { a.e. }\left(x^{\prime}, y_{3}\right) \in \widetilde{\Omega}_{\varepsilon} .
$$

Let us introduce some notation which will be useful in the following: for a vectorial function $v=\left(v^{\prime}, v_{3}\right)$ (and, analogously, for a scalar function $w$ ), associated to the change of variables (1.6), we introduce the operators $\mathbb{D}_{\mathcal{\varepsilon}}, D_{\varepsilon}, \operatorname{div}_{\varepsilon}$ and $\nabla_{\varepsilon}$, defined by

$$
\begin{gathered}
\mathbb{D}_{\varepsilon}[v]=\frac{1}{2}\left(D_{\varepsilon} v+D_{\varepsilon}^{t} v\right), \quad\left|\mathbb{D}_{\varepsilon}[v]\right|^{2}=\mathbb{D}_{\varepsilon}[v]: \mathbb{D}_{\varepsilon}[v] \\
\left(D_{\varepsilon} v\right)_{i, j}=\partial_{x_{j}} v_{i} \text { for } i=1,2,3, j=1,2, \quad\left(D_{\varepsilon} v\right)_{i, 3}=\frac{1}{\varepsilon} \partial_{y_{3}} v_{i} \text { for } i=1,2,3, \\
\operatorname{div}_{\varepsilon} v=\operatorname{div}_{x^{\prime}} v^{\prime}+\frac{1}{\varepsilon} \partial_{y_{3}} v_{3}, \quad \nabla_{\varepsilon} w=\left(\nabla_{x^{\prime}} w, \frac{1}{\varepsilon} \partial_{y_{3}} w\right)^{t} .
\end{gathered}
$$

We consider the space

$$
V\left(\widetilde{\Omega}_{\varepsilon}\right)=\left\{\tilde{v} \in\left(H_{0}^{1}\left(\widetilde{\Omega}_{\varepsilon}\right)\right)^{3} \mid \operatorname{div}_{\varepsilon} \tilde{v}=0 \text { in } \widetilde{\Omega}_{\varepsilon}\right\}
$$

and for $\tilde{u}, \tilde{v} \in V\left(\widetilde{\Omega}_{\varepsilon}\right)$, we introduce

$$
a_{\varepsilon}(\tilde{u}, \tilde{v})=2 \mu \int_{\widetilde{\Omega}_{\varepsilon}} \mathbb{D}_{\varepsilon}[\tilde{u}]: \mathbb{D}_{\varepsilon}[\tilde{v}] d x^{\prime} d y_{3},
$$




$$
j_{\varepsilon}(\tilde{v})=\sqrt{2} g \varepsilon \int_{\widetilde{\Omega}_{\varepsilon}}\left|\mathbb{D}_{\mathcal{\varepsilon}}[\tilde{v}]\right| d x^{\prime} d y_{3}, \quad(\tilde{u}, \tilde{v})_{\widetilde{\Omega}_{\varepsilon}}=\int_{\widetilde{\Omega}_{\varepsilon}} \tilde{u} \cdot \tilde{v} d x^{\prime} d y_{3} .
$$

Using the transformation (1.6), the variational inequality (1.5) can be rewritten as:

Find $\tilde{u}_{\varepsilon} \in V\left(\widetilde{\Omega}_{\varepsilon}\right)$ such that

$$
a_{\varepsilon}\left(\tilde{u}_{\varepsilon}, \tilde{v}-\tilde{u}_{\varepsilon}\right)+j_{\varepsilon}(\tilde{v})-j_{\varepsilon}\left(\tilde{u}_{\varepsilon}\right) \geq\left(f, \tilde{v}-\tilde{u}_{\varepsilon}\right)_{\tilde{\Omega}_{\varepsilon}}, \quad \forall \tilde{v} \in V\left(\widetilde{\Omega}_{\varepsilon}\right) .
$$

We start by obtaining some a priori estimates for $\tilde{u}_{\varepsilon}$, stated in the next lemma.

Lemma 1. There exists a constant $C$ independent of $\varepsilon$, such that if $\tilde{u}_{\varepsilon} \in\left(H_{0}^{1}\left(\widetilde{\Omega}_{\varepsilon}\right)\right)^{3}$ is the solution of problem (1.7), one has

$$
\begin{gathered}
\left\|\tilde{u}_{\varepsilon}\right\|_{\left(L^{2}\left(\widetilde{\Omega}_{\varepsilon}\right)\right)^{3}} \leq C \varepsilon^{2}, \quad\left\|\mathbb{D}_{\varepsilon}\left[\tilde{u}_{\varepsilon}\right]\right\|_{\left(L^{2}\left(\widetilde{\Omega}_{\varepsilon}\right)\right)^{3 \times 3}} \leq C \varepsilon, \\
\left\|D_{\varepsilon} \tilde{u}_{\varepsilon}\right\|_{\left(L^{2}\left(\widetilde{\Omega}_{\varepsilon}\right)\right)^{3 \times 3}} \leq C \varepsilon .
\end{gathered}
$$

We extend the velocity $\tilde{u}_{\varepsilon}$ by zero to the $\Omega \backslash \widetilde{\Omega}_{\varepsilon}$ and denote the extension by the same symbol. Obviously, estimates (1.8)-(1.9) remain valid for the extended function and the extension is divergence free too. According to this extension, problem (1.7) can be written as:

$$
\begin{aligned}
& 2 \mu \int_{\Omega} \mathbb{D}_{\varepsilon}\left[\tilde{u}_{\varepsilon}\right]: \mathbb{D}_{\varepsilon}\left[\tilde{v}-\tilde{u}_{\varepsilon}\right] d x^{\prime} d y_{3}+\sqrt{2} g \varepsilon \int_{\Omega}\left|\mathbb{D}_{\varepsilon}[\tilde{v}]\right| d x^{\prime} d y_{3} \\
& -\sqrt{2} g \varepsilon \int_{\Omega}\left|\mathbb{D}_{\varepsilon}\left[\tilde{u}_{\varepsilon}\right]\right| d x^{\prime} d y_{3} \geq \int_{\Omega} f \cdot\left(\tilde{v}-\tilde{u}_{\varepsilon}\right) d x^{\prime} d y_{3},
\end{aligned}
$$

for every $\tilde{v}$ that is the extension by zero to the whole $\Omega$ of a function in $\left(H_{0}^{1}\left(\widetilde{\Omega}_{\mathcal{E}}\right)\right)^{3}$.

Our main result is the following theorem:

Theorem 1. Let $\tilde{u}_{\varepsilon}$ be the solution of problem (1.10). There exists $\hat{u} \in L^{2}\left(\omega ; H_{\sharp}^{1}(Y)^{3}\right)$ (here “ $\sharp$ denotes $Y^{\prime}$-periodicity), such that

$$
\frac{\tilde{u}_{\varepsilon}}{\varepsilon^{2}} \rightarrow \int_{Y^{\prime}} \hat{u} d y^{\prime} \text { in } L^{2}(\Omega)
$$

$\int_{Y^{\prime}} \hat{u}_{3} d y^{\prime}=0, \quad \hat{u}=0$ on $\omega \times Y_{s}, \quad \hat{u}=0$ on $y_{3}=\{0,1\}, \quad \operatorname{div}_{y} \hat{u}=0$ in $\omega \times Y$,

$$
\operatorname{div}_{x^{\prime}}\left(\int_{Y} \hat{u}^{\prime}\left(x^{\prime}, y\right) d y\right)=0 \text { in } \omega, \quad\left(\int_{Y} \hat{u}^{\prime}\left(x^{\prime}, y\right) d y\right) \cdot n=0 \text { on } \partial \omega,
$$

and $\hat{u}$ is the unique solution of the limit problem

$$
\begin{aligned}
& 2 \mu \int_{\omega \times Y} \mathbb{D}_{y}[\hat{u}]:\left(\mathbb{D}_{y}[\tilde{v}]-\mathbb{D}_{y}[\hat{u}]\right) d x^{\prime} d y+\sqrt{2} g \int_{\omega \times Y}\left|\mathbb{D}_{y}[\tilde{v}]\right| d x^{\prime} d y \\
& -\sqrt{2} g \int_{\omega \times Y}\left|\mathbb{D}_{y}[\hat{u}]\right| d x^{\prime} d y \geq \int_{\omega \times Y} f^{\prime} \cdot\left(\tilde{v}^{\prime}-\hat{u}^{\prime}\right) d x^{\prime} d y,
\end{aligned}
$$

for every $\tilde{v} \in L^{2}\left(\omega ; H_{\sharp}^{1}(Y)^{3}\right)$ such that $\tilde{v}\left(x^{\prime}, y\right)=0$ in $\omega \times Y_{s}, \quad \operatorname{div}_{y} \tilde{v}=0$ in $\omega \times Y$, 


$$
\operatorname{div}_{x^{\prime}}\left(\int_{Y} \tilde{v}^{\prime}\left(x^{\prime}, y\right) d y\right)=0 \text { in } \omega, \quad\left(\int_{Y} \tilde{v}^{\prime}\left(x^{\prime}, y\right) d y\right) \cdot n=0 \text { on } \partial \omega .
$$

Proof: First step A priori estimates

The change of variable (1.6) does not provide the information we need about the behavior of $\tilde{u}_{\varepsilon}$ in the microstructure associated to $\widetilde{\Omega}_{\varepsilon}$. To solve this difficulty, we use an adaptation of the unfolding method from [CiEtAl08]. In order to apply the unfolding method, we will need the following notation: for $k^{\prime} \in \mathbb{Z}^{2}$, we define $\kappa: \mathbb{R}^{2} \rightarrow \mathbb{Z}^{2}$ by

$$
\kappa\left(x^{\prime}\right)=k^{\prime} \Longleftrightarrow x^{\prime} \in Y_{k^{\prime}, 1}^{\prime} .
$$

Remark that $\kappa$ is well defined up to a set of zero measure in $\mathbb{R}^{2}$ (the set $\cup_{k^{\prime} \in \mathbb{Z}^{2}} \partial Y_{k^{\prime}, 1}^{\prime}$ ). Moreover, for every $\varepsilon>0$, we have

$$
\kappa\left(\frac{x^{\prime}}{\varepsilon}\right)=k^{\prime} \Longleftrightarrow x^{\prime} \in Y_{k^{\prime}, \varepsilon}^{\prime} .
$$

According to the adaptation introduced in [AS17] of the unfolding method, we divide the domain $\Omega$ in rectangular cuboids of lateral lengths $\varepsilon$ and vertical length 1. For this purpose, given $\tilde{u}_{\varepsilon} \in\left(H_{0}^{1}(\Omega)\right)^{3}$, we define $\hat{u}_{\varepsilon}$ by

$$
\hat{u}_{\varepsilon}\left(x^{\prime}, y\right)=\tilde{u}_{\varepsilon}\left(\varepsilon \kappa\left(\frac{x^{\prime}}{\varepsilon}\right)+\varepsilon y^{\prime}, y_{3}\right), \text { a.e. }\left(x^{\prime}, y\right) \in \omega \times Y,
$$

where the function $\kappa$ is defined in (1.12).

For $k^{\prime} \in \mathscr{K}_{\varepsilon}$, the restriction of $\hat{u}_{\varepsilon}$ to $Y_{k^{\prime}, \varepsilon}^{\prime} \times Y$ does not depend on $x^{\prime}$, whereas as a function of $y$ it is obtained from $\tilde{u}_{\varepsilon}$ by using the change of variables

$$
y^{\prime}=\frac{x^{\prime}-\varepsilon k^{\prime}}{\varepsilon}
$$

which transforms $Y_{k^{\prime}, \varepsilon}$ into $Y$.

We can obtain a priori estimates for the sequence $\hat{u}_{\varepsilon}$. There exists a constant $C$ independent of $\varepsilon$, such that $\hat{u}_{\varepsilon}$ defined by (1.13) satisfies

$$
\begin{gathered}
\left\|\mathbb{D}_{y}\left[\hat{u}_{\varepsilon}\right]\right\|_{\left(L^{2}(\omega \times Y)\right)^{3 \times 3}} \leq C \varepsilon^{2}, \\
\left\|\hat{u}_{\varepsilon}\right\|_{\left(L^{2}(\omega \times Y)\right)^{3}} \leq C \varepsilon^{2} .
\end{gathered}
$$

Second step Convergence results

As in [AS17], we can obtain compactness results for the sequences $\tilde{u}_{\varepsilon}$ and $\hat{u}_{\varepsilon}$ satisfying the a priori estimates given before.

For a subsequence of $\varepsilon$ still denoted by $\varepsilon$, there exist $\tilde{u} \in H^{1}\left(0,1 ; L^{2}(\omega)^{3}\right)$, where $\tilde{u}_{3}=0$ and $\tilde{u}=0$ on $y_{3}=\{0,1\}, \hat{u} \in L^{2}\left(\omega ; H_{\sharp}^{1}(Y)^{3}\right)$ (“\#” denotes $Y^{\prime}$-periodicity), with $\hat{u}=0$ on $\omega \times Y_{S}$ and $\hat{u}=0$ on $y_{3}=\{0,1\}$ such that $\int_{Y} \hat{u}\left(x^{\prime}, y\right) d y=\int_{0}^{1} \tilde{u}\left(x^{\prime}, y_{3}\right) d y_{3}$ with $\int_{Y} \hat{u}_{3} d y=0$, such that 


$$
\begin{gathered}
\frac{\tilde{u}_{\varepsilon}}{\varepsilon^{2}} \rightarrow\left(\tilde{u}^{\prime}, 0\right) \text { in } H^{1}\left(0,1 ; L^{2}(\omega)^{3}\right) \\
\frac{\hat{u}_{\varepsilon}}{\varepsilon^{2}} \rightarrow \hat{u} \text { in } L^{2}\left(\omega ; H^{1}(Y)^{3}\right) \\
\operatorname{div}_{y} \hat{u}=0 \text { in } \omega \times Y \\
\operatorname{div}_{x^{\prime}}\left(\int_{0}^{1} \tilde{u}^{\prime}\left(x^{\prime}, y_{3}\right) d y_{3}\right)=0 \text { in } \omega, \quad\left(\int_{0}^{1} \tilde{u}^{\prime}\left(x^{\prime}, y_{3}\right) d y_{3}\right) \cdot n=0 \text { on } \partial \omega \\
\operatorname{div}_{x^{\prime}}\left(\int_{Y} \hat{u}^{\prime}\left(x^{\prime}, y\right) d y\right)=0 \text { in } \omega, \quad\left(\int_{Y} \hat{u}^{\prime}\left(x^{\prime}, y\right) d y\right) \cdot n=0 \text { on } \partial \omega
\end{gathered}
$$

Third Step Passage to the limit

By using (1.13), we first transform the variational inequality (1.10) in a variational inequality stated in the domain $\omega \times Y$. Then, by choosing suitable test functions, we pass to the limit $\varepsilon \rightarrow 0$. By using convergences (1.16), (1.17), (1.18) we find the limit problem (1.11). The uniqueness of the solution $\hat{u}$ of problem (1.11) is proved by contradiction.

\subsection{Conclusions and perspectives}

By using dimension reduction and homogenization techniques, we studied the limiting behavior of the velocity for a nonlinear viscoplastic Bingham flow with small yield stress $g \varepsilon$, in a thin porous medium of small height $\varepsilon$ and for which the relative dimension of the pores is $\varepsilon$. We found in Theorem 1 the limit problem (1.11), in which both effects of a nonlinear Darcy law and a lower-dimensional Binghamlike law appear. Indeed, as in [LiEtAl81](see also [BuEtAl17]), problem (1.11) can be written as a nonlinear Darcy law set in the domain $\omega$. The third component of the velocity of filtration appearing in the nonlinear Darcy law equals zero and this phenomenon corresponds precisely to a two-dimensional Bingham-like law (see [BuEtA104]).

In the forthcoming work [AnEtAl] we study the cases of thin porous media those periodicity parameter is $a_{\varepsilon}$ instead of $\varepsilon$. Different cases are analysed, following the ratio between the height $\varepsilon$ of the porous media and the relative dimension $a_{\varepsilon}$ of the periodically distributed pores. Moreover, we consider the more involved case in which the convergence of the pressure of the flow is also studied.

\section{Acknowledgments}

María Anguiano has been supported by Junta de Andalucía (Spain), Proyecto de Excelencia P12-FQM-2466. 


\section{References}

[Zh08] Zhengan, Y., and Hongxing, Z.: Homogenization of a stationary Navier-Stokes flow in porous medium with thin film. Acta Mathematica Scientia, 28B(4), 963-974 (2008).

[FaEtAl16] Fabricius, J., Hellström, J.G. I., Lundström, T.S., Miroshnikova E., and Wall, P.: Darcy's Law for Flow in a Periodic Thin Porous Medium Confined Between Two Parallel Plates. Transp. Porous Med., 115, 473-493 (2016).

[AS18] Anguiano, M., and Suárez-Grau, F.J.: The transition between the Navier-Stokes equations to the Darcy equation in a thin porous medium. Mediterr. J. Math., 15:45 (2018).

[AS17] Anguiano, M., and Suárez-Grau, F.J.: Homogenization of an incompressible nonNewtonian flow through a thin porous medium. Z. Angew. Math. Phys., 68:45 (2017).

[CiEtA108] Cioranescu, D., Damlamian, A., and Griso, G.: The periodic Unfolding Method in Homogenization. SIAM J. Math. Anal., 40, No. 4, 1585-1620 (2008).

[An17] Anguiano, M.: Darcy's laws for non-stationary viscous fluid flow in a thin porous medium. Math. Meth. Appl. Sci., 40, No. 8, 2878-2895 (2017).

[An217] Anguiano, M.: On the non-stationary non-Newtonian flow through a thin porous medium. ZAMM-Z. Angew. Math. Mech., 97, No. 8, 895-915 (2017).

[Gr04] Griso, G.: Asymptotic behavior of a crane.C.R.Acad.Sci. Paris, Ser. I, 338, No. 3, 261-266.

[GrEtA117] Griso, G., Migunova, A., and Orlik, J.: Asymptotic analysis for domains separated by a thin layer made of periodic vertical beams. Journal of Elasticity, 128, 291-331 (2017).

[LiEtAl81] Lions, J.L., and Sánchez-Palencia E.: Écoulement d'un fluide viscoplastique de Bingham dans un milieu poreux. J. Math. Pures Appl., 60, 341-360 (1981).

[BoEtA193] Bourgeat, A., and Mikelić, A.: A note on homogenization of Bingham flow through a porous medium. J. Math. Pures Appl., 72, 405-414 (1993).

[BuEtAl13] Bunoiu R., Cardone G., and Perugia C.: Unfolding Method for the Homogenization of Bingham flow. In Modelling and Simulation in Fluid Dynamics in Porous Media, Series: Springer Proceedings in Mathematics \& Statistics, Vol. 28 (2013), pp. 109123.

[BuEtAl17] Bunoiu, R., and Cardone, G.: Bingham Flow in Porous Media with Obstacles of Different Size. Mathematical Methods in the Applied Sciences(MMAS), 40, No. 12, 4514-4528 (2017).

[BuEtA103] Bunoiu, R., and Kesavan, S.: Fluide de Bingham dans une couche mince, In Annals of the University of Craiova. Math. Comp. Sci. series 30, (2003), pp 1-9.

[BuEtA104] Bunoiu, R., and Kesavan, S.: Asymptotic behaviour of a Bingham fluid in thin layers. Journal of Mathematical Analysis and Applications, 293, No. 2, 405-418 (2004).

[BuEtAl18] Bunoiu, R., Gaudiello A., and Leopardi A.: Asymptotic Analysis of a Bingham Fluid in a Thin T-like Shaped Structure. Journal de Mathématiques Pures et Appliquées, https://doi.org/10.1016/j.matpur.2018.01.001.

[LiEtA190] Liu K.F., and Mei, C.C.: Approximate equations for the slow spreading of a thin sheet of Bingham plastic fluid. Phys. Fluids, A 2, 30-36 (1990).

[DuEtA172] Duvaut, G., and Lions, J.L.: Les inéquations en mécanique et en physique. Dunod, Paris (1972).

[AnEtAl] Anguiano, M., and Bunoiu, R.: Homogenization of Bingham flow in thin porous media. In preparation. 